\title{
An Unusual Complication of Ureteral Stone: Massive Gastric Dilatation and Duodenal Obstruction Due to Giant Hydronephrosis
}

\author{
Mehmet Ali Karagoz, Mucahit Kabar, Arif Demirbas, Onur Telli* \\ Ankara Training and Research Hospital, Ankara, Turkey \\ *Corresponding author: onurtelli@yahoo.com
}

Received August 18, 2013; Revised August 28; Accepted September 12, 2013

\begin{abstract}
A 42 year old man, was admitted to emergency service who suffered from nausea and vomitting for two days. In his physical examination the right side abdomen was severe distended, that had been lasting for a month. Distension was tender and cystic tough mass lesion was palbable. Plain abdominal radiography showed a $3 \mathrm{~cm}$ radiopaque shadow in the pelvic area. Ct scan revealed massive gastric dilatation and duodenal obstruction due to giant hydronephrosis with the ureteric stone placing on the right lower ureter with a dimension of 3,5 to $3 \mathrm{~cm}$. Nasogastrical decompression and right percutaneus nephrostomy catheter was performed, $5000 \mathrm{ml}$ of gastric fluid and $5300 \mathrm{ml}$ of nephrostomy catheter drainage was measured in the first six hours. 99m Tc-dimercaptosuccinic acid (DMSA) scan showed non-functioning right kidney and the patient finally underwent open simple nephroureterectomy. To the best of our knowledge the present patient represents one of the rarest complication of giant hydronephrosis cases reported to date.
\end{abstract}

Keywords: giant hydronephrosis, gastric dilatation, ureteral stone

Cite This Article: Mehmet Ali Karagoz, Mucahit Kabar, Arif Demirbas, and Onur Telli, “An Unusual Complication of Ureteral Stone: Massive Gastric Dilatation and Duodenal Obstruction due to Giant Hydronephrosis.” American Journal of Clinical Medicine Research 1, no. 4 (2013): 51-53. doi: 10.12691/ajcmr1-4-2.

\section{Introduction}

Hydronephrosis and flank pain due to ureteral stones is an expect antclinical outcome and flank pain it's the most frequently appealing reason as a cause hydronephrosis. However, giant hydronephrosis, duodenal obstruction and massive gastric dilatation is a very rare and unusual complication of ureteral stone. According to Tombari et al, approximately 2000 cases of gianth ydronephrosis have been reported, since its first description in 1968 [1]. Giant hydronephrosis defined as the collecting system of kidney containing more than one liter of urine or \% 1.6 of body weight or a kidney occupying a hemiabdomen [2,3]. We present a case report with duodenal obstruction and massive gastric dilatation caused by ureteral stone and giant hydronephrosis, without no flank pain and with abnormal complaints.

\section{Case Report}

A 42 year old man, was admitted to emergency service who suffered from nausea and vomitting for two days. Patient has no known disease and medical history. In his physical examination the right side abdomen was severe distended, that had been lasting for a month. Distension was tender and cystic tough mass lesion was palbable.
Bowel sounds were hyperactive. The patient was not constipated or anuric. Body temperature was $37.8^{\circ} \mathrm{C}$. In urinalysis microscopic hematuria and pyuria was detected. In hemogram analysis leukocytosis was detected. The serum creatine level was $1,3 \mathrm{mg} / \mathrm{dl}$. Abdominal ultrasound evaluation did not differantiate an hydnephrotic kidney or an abdominal mass lesion either its origin. Because of the gastric complaints iv opaques Ct scan was performed. And it revealed massive gastric dilatation and duodenal obstruction due to giant hydronephrosis (grade V) with lower ureteral Stone (Figure 1). Also the kidney, ureter, and bladder X-Ray (KUB) evaluation showed the round ureteric stone placing on the right side pelvic bone with a dimension of 3,5 to $3 \mathrm{~cm}$. Following that nasogastrical decompression and right percutaneus nephrostomy catheter was performed, $5000 \mathrm{ml}$ of gastric fluid and 5300 $\mathrm{ml}$ of nephrostomy catheter drainage was measured in the first six hours. Cytology and urine culture was also sent from nephrostomy catheter drainage. Thickness of renal cortex was $2 \mathrm{~mm}$ or immeasureble . 99m Tcdimercaptosuccinic acid (DMSA) scan showed nonfunctioning right kidney and the patient finally underwent open simple nephroureterectomy using flank incision and the ureteral stone was taken out (Figure 2). The kidney tissue were very adhesive to liver and other organs. At postoperative follow up no complication or blood transfusion were needed. Postoperative first day internal 
urinary catheter, and the second day drainage tube was taken off.

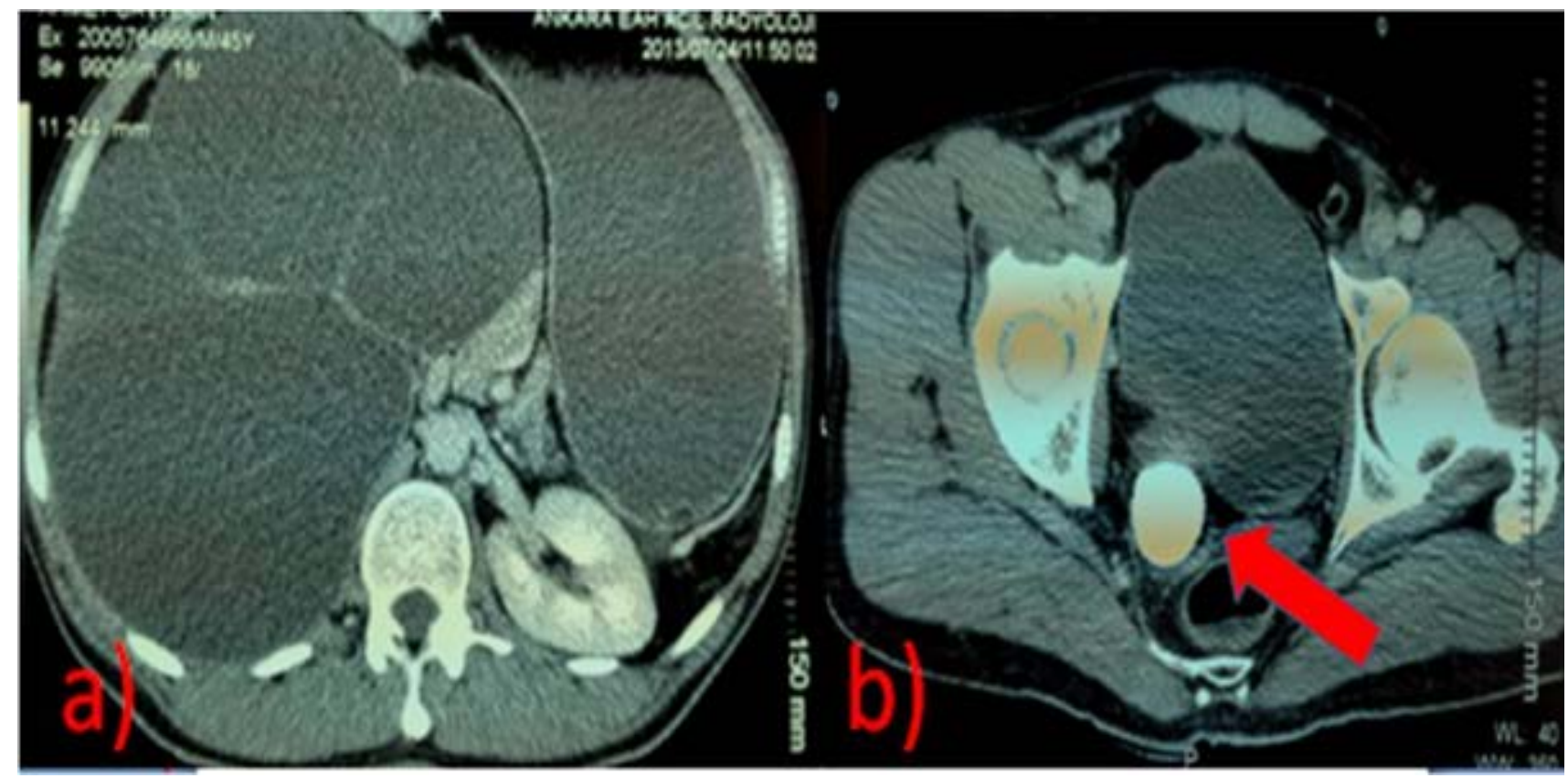

Figure 1. Abdominal computed tomography a) Massive gastric dilatation and duodenal obstruction due to giant hydronephrosis b) 3,5x3cm giant ureteral stone

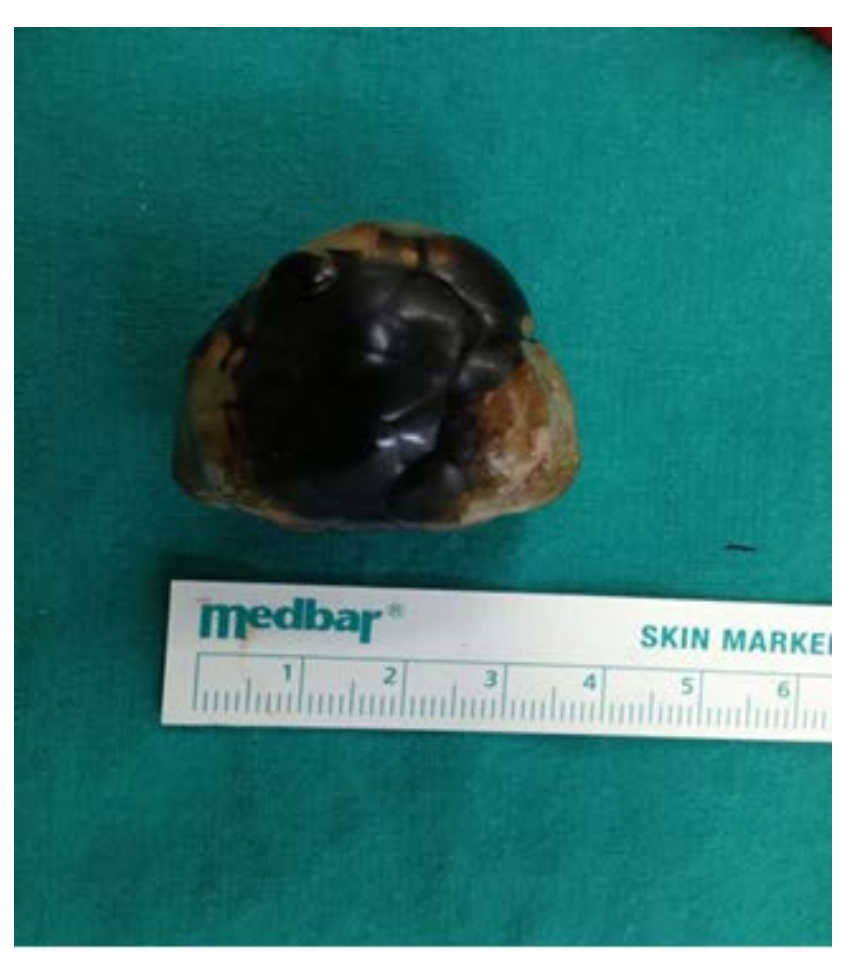

Figure 2. Giant ureteral stone $[3,5 \times 3 \mathrm{~cm}]$

\section{Discussion}

Mild hydronephrosis caused by ureteral stones is common and we experience often in our daily practice. Patients may refer with flank pain, disuria, hematuria, acute abdomen and recurrent urinary tract infections [4,5]. Most cases of giant hydronephrosis results from the devolopment of a lesion in upper urinary tract which is thought to be because of lower urinary tract lesion as were ported in our case like a huge ureteral stone. However, giant hydronephrosisis a very rare complication and according to Morimutsu et al, uretero pelvic junction (UPJ) narrowing, ureteral stones, congenital ureteral narrowing and ureteral or UPJ tumors in elder ages are the most common reasons of giant hydronephrosis [6]. Duodenal obstruction and gastric dilatation in adults are also rare. In literature there are limited reported cases of intestinal obstruction due to giant hydronephrosis $[7,8]$. This case seems to be the first that massive gastric dilatation and duodenal obstruction caused by giant hydronephrosis due to ureteral stone. In our case primarily, we assumed that the patient might had an undiagnosed primary obstructive megaureter (POM) because of the dilated hydro ureter until the lower segment and results as a giant ureteral stone. Megaureter with a adynamic segment in the distal ureter generally, presents symptoms in the third or fourth decades of life. It is also usually unilateral; however, in $15-25 \%$ of cases it can be bilateral. Because the distal ureter is adynamic, it is obstructed functionally. Normal upper ureter dilatation occurs secondary to functional obstruction [9].

\section{Conclusion}

Alternatives for ureteral stones treatment vary from endourologic techniques to laparoscopic surgery and open surgery. Extracorporeal shock wave lithotripsy(ESWL) is also an non-invasive alternative for selected cases. However, giant hydronephrosis is usually related with impaired renal function and cortical thickness loss is determined in radiological evaluations. DMSA scan was performed to this patient and revealed that no cortical up take of radionuclide substance of the hydronephrotic kidney. Hoffman stated that nephrectomy is often the only therapy for giant hydronephrosis because there is no feasible prospect of improvement in renal function especially if the function of the contralateral kidney is normal [10]. 


\section{References}

[1] Tombari AA, Power FR, Harper JM et al. Giant Hydronephrosis: a case report with the review of literature. J Urol 1968; 473-6.

[2] Stirling WC, Massive hydronephrosis complicated by hydroureter. J Urol 42: 520-533 1939.

[3] Chiang PH, Chen MT, Chou YH, Chiang CP, Huang $\mathrm{CH}$, Chien $\mathrm{CH}$. Giant hydronephrosis: report of 4 cases with review of the literature. J FormosMedAssoc. 1990;89(9):811-7.

[4] Yapanolu T, Alper F, Ozbey, et al. Giant hydronephrosis mimicking an intraabdominal mass. Turk J Med Sci.2007;37:177-9.

[5] Shah SA, Ranka P, Dodiya S, et al. Gian thydronephrosis: What is the ideal treatment? Indian Journal of Urology. 2004;20(2):118.
[6] Morimitsu H, Sakaguchi M, Suzu H, et al. Giant hydronephrosis: 2 case reports and a review of 373 cases in the literature. Nishinihon J Urol 1990;52:761-766.

[7] LYGONIS CS. Intestinal obstruction caused by hydronephrosis. PostgradMed J. 1961 Apr;37:213.

[8] Ryushi S, Tetsuya S, Kuniyasu T, et al. Gian thydronephrosis due to a ureteral stone, and elevated serum levels of CA 19-9. Intern Med. 1999 Nov;38(11):887-91.

[9] Hemal AK, Ansari MS, Doddamani D, Gupta NP. Symptomatic and complicated adult and adolescent primary obstructive megaureter-indications for surgery: analysis, outcome, and followup. Urology. 2003 Apr;61(4):703-7; discussion 707.

[10] Hoffman HA. Massive hydronephrosis. J Urol. 1948;59(5):78494. 